\title{
Polar Cosine-Sine Transform for Image Representation
}

\author{
Kezheng Sun ${ }^{1+}$ and Lijuan Tang ${ }^{1,2}$ \\ ${ }^{1}$ School of Info., Vocational Col. of Bus., China \\ ${ }^{2}$ School of Info. and Elec. Eng., China Univ. of Mining and Technology, China
}

\begin{abstract}
Since Polar Harmonic Transforms (PHTs) have been introduced, they are widely used in image analysis and pattern recognition with computation of the kernel is exceedingly briefness. However, PHTs always have weakness of instability at high orders of moments. What's worse? PCET encounters this undesirable situation even at low orders. This paper presents the Polar Cosine-Sine Transform (PCST) using a new radial kernel based on Cosine-Sine functions to achieve all-rights stability. First, we analyze the radial kernel and the reason of instability of the traditional PCET. Second, we structure a new radial kernel using Cosine-Sine functions based on analysis of PCET. Finally, PCST has been introduced. The experimental result shows that the proposed method holds rotation invariance and the stability of PCST is superior to PHTs in terms of image representation capability when high-order moments are concerned.
\end{abstract}

Keywords: Orthogonal moments, Polar harmonic transforms, Rotation invariants, Polar cosine-sine transform.

\section{Introduction}

As we all know, invariance of moments is a basic requirement in many image-processing analysis and applications. For decades, individuals have introduced so many orthogonal rotation-invariant moments and transforms. For example, Zernike moments(ZM) [1], pseudo-Zernike moments(PZM) [2], orthogonal FourierMellin moments(OFMM) [3], and pseudo Jacobi-Fourier moments(PJFM) [4]. These are the Jacobi polynomial-based radial kernels methods. For these moments the above mentioned, orthogonality have been achieved and they are rotation-invariant. However, despite its popularity, the radial kernels involves computation of complicated factorial terms, which inevitably cause a lot of time and limits their practical usefulness. 2010, Pew-Thian Yap et al. [5] introduced a set of 2D transforms called Polar Harmonic Transforms (PHTs). They have been successfully applied in a variety of contexts including, but not limited to image watermarking, pattern recognition [5], fingerprint classification [6], etc. However, PHTs always have disadvantage of instability when high-order moments are concerned. PCET first encounters this undesirable situation even at low order. This shortcoming greatly limited the applications of PHTs.

For the last few years, several algorithms have been proposed for improving PHTs. C.Singh et al. [7] proposed a computational framework based on numerical integration approach that reduces the geometric error and the numerical integration error simultaneously. Wo Yan et al. [8] utilized partition of polar coordinates and the inherent recurrence relations about complex exponential and trigonometric functions to improve accuracy and numerical stability. Thai V. Hoang et al. [9] introduced three classes of rotation-invariant orthogonal moments by generalizing PCET, PCT, and PST that use harmonic functions in their radial kernels. In addition, each member of one of these classes has distinctive properties that depend on the value of a parameter, making them more suitable for some particular applications. However, it's impractical to generalize the PHTs in some cases through three formulas.

\footnotetext{
+ Corresponding author. Tel.: + +86-13515202135.

E-mail address: 528106tyhj@163.com.
} 
Based on this observation, this paper presents the Polar Cosine-Sine Transforms (PCST) using a new radial kernel based on Cosine-Sine functions to achieve stability. The proposed radial kernels are excelled the traditional methods with the increase of the quantity of moments. Therefore, the stability of PCST is superior to PHTs, in terms of image representation capability. In addition, PCST hold rotation invariance, which is an important property for pattern recognition.

\section{Definition of PHTs}

Pew-Thian Yap et al. [5] introduced a set of 2D transforms called Polar Harmonic Transforms (PHTs), including Polar Complex Exponential Transform (PCET), Polar Cosine Transform (PCT) and Polar Sine Transform (PST):

$$
\begin{gathered}
M_{n l}=\Omega_{n} \int_{0}^{2 \pi} \int_{0}^{1}\left[H_{n l}(r, \theta)\right]^{*} f(r, \theta) r d r d \theta \\
=\Omega_{n} \int_{0}^{2 \pi} \int_{0}^{1}\left[R_{n}(r, \theta) e^{i l \theta}\right]^{*} f(r, \theta) r d r d \theta
\end{gathered}
$$

where [.]* denotes the complex conjugate.

Orthogonality of complex exponential function and trigonometric function has been confirmed in Mathematics, and these functions have the advantages of simple computation. Consequently, characters exploit these functions to construct moments.

It is not difficult to find from definition of PHTs that the total number of PCET is $\left(1+2 n_{\max }\right)\left(1+2 l_{\max }\right)$, but PCT and PST have less number of moments, which are $\left(1+n_{\max }\right)\left(1+2 l_{\max }\right)$ and $n_{\max }\left(1+2 l_{\max }\right)$, respectively. PHTs are Orthogonal rotation-invariant transforms.

The kernel of PHTs is the set of orthogonal complex functions defined inside a unit circle over the polar coordinate space. In fact, however, images are defined on a discrete domain. Consequently, (1) can be written in Cartesian coordinates as:

$$
M_{n l}=\frac{1}{\pi} \iint_{x^{2}+y^{2} \leq 1}\left[H_{n l}^{\prime}(x, y)\right]^{*} f^{\prime}(x, y) d x d y,
$$

where $H_{n l}^{\prime}(x, y)=H_{n l}^{\prime}(r \cos \theta, r \sin \theta) \equiv H_{n l}(r, \theta)$ and $f^{\prime}(x, y)=f(r \cos \theta, r \sin \theta) \equiv f(r, \theta)$

A detailed discussion about discretization and mapping will be found in [10].

\section{Polar Cosine-Sine Transform}

Considering the underdeveloped performance the above analyzed, we introduces a new transform namely Polar Cosine-Sine Transform, which is based on Cosine-Sine functions as radial kernel. The number and distribution of zeros of the proposed radial kernel are excelled the traditional PHTs. Consequently, PCST is superior to PHTs when high-order moments are concerned in terms of image representation capability.

\subsection{Definition of PCST}

The Polar Cosine-Sine Transform of order $n$ with repletion $l$ is defined as

$$
M_{n l}=\frac{1}{\pi} \int_{0}^{1} \int_{0}^{2 \pi}\left[H_{n l}(r, \theta)\right]^{*} f(r, \theta) r d r d \theta,
$$

where $|n|=|l|=0,1,2, \ldots, \infty$.

The basis $H_{n l}(r, \theta)$ can be decomposed into radial and circular components:

$$
H_{n l}(r, \theta)=R_{n}^{c s}(r) e^{i l \theta}
$$

with the radial kernel being a Cosine-Sine in the radial direction defined as

$$
R_{n}^{c s}(r)=a \cos \left(\pi n r^{2}\right)+b i \sin \left(\pi n r^{2}\right)
$$

where $\frac{a^{2}+b^{2}}{2}=1 \& \& a \neq 0 \& \& b \neq 0$. 
Obviously, then satisfying orthogonality condition:

$$
\int_{0}^{1} R_{n}^{c s}(r) R_{n^{\prime}}^{c s^{*}}(r) r d r=\frac{1}{2} \delta_{n n^{\prime}}
$$

And, hence:

$$
\begin{gathered}
\left\langle H_{n l}, H_{n^{\prime} l^{\prime}}\right\rangle=\iint_{\Omega} H_{n l}(x, y) H_{n^{\prime} l^{\prime}}^{*}(x, y) d x d y \\
=\int_{0}^{1} \int_{0}^{2 \pi} R_{n}(r) A_{l}(\theta)\left[R_{n^{\prime}}(r) A_{l^{\prime}}(\theta)\right]^{*} r d r d \theta \\
=\int_{0}^{1} R_{n}(r) R_{n^{\prime}}^{*}(r) r d r \int_{0}^{2 \pi} A_{l}(\theta) A_{l^{\prime}}^{*}(\theta) d \theta \\
=\pi \delta_{n n^{\prime}} \delta_{l l^{\prime}}
\end{gathered}
$$

In fact, the factor $1 / \pi$ of (3) is due to the norm of the kernels. We just need to make small changes of kernels, it means:

$$
\hat{H}_{n l}(r, \theta)=\frac{1}{\sqrt{\pi}} H_{n l}(r, \theta),
$$

And hence

$$
\left\langle H_{n l}, H_{n^{\prime} l^{\prime}}\right\rangle=\iint_{\Omega} H_{n l}(x, y) H_{n^{\prime} l^{\prime}}^{*}(x, y) d x d y=\delta_{n n^{\prime}} \delta_{l l^{\prime}} .
$$

Rotation invariance is the one of most paramount properties of orthogonal transforms. If image $f(r, \theta)$ is rotated through a counter clockwise angle $\alpha$ to become $f^{\alpha}(r, \theta)=f(r, \theta-\alpha)$ with the PCST coefficients:

$$
\begin{gathered}
M_{n l}^{\alpha}=\frac{1}{\pi} \int_{0}^{1} \int_{0}^{2 \pi}\left[H_{n l}(r, \theta)\right]^{*} f^{\alpha}(r, \theta) r d r d \theta \\
=\frac{1}{\pi} \int_{0}^{1} \int_{0}^{2 \pi}\left[R_{n}^{c s}(r) e^{i l \theta}\right]^{*} f(r, \theta-\alpha) r d r d \theta \\
\begin{array}{c}
\varphi=\theta-\alpha \\
=
\end{array} \frac{1}{\pi} \int_{0}^{1} \int_{0}^{2 \pi}\left[R_{n}^{c s}(r) e^{i l(\varphi+\alpha)}\right]^{*} f(r, \varphi) r d r d \varphi \\
=\frac{1}{\pi} \int_{0}^{1} \int_{0}^{2 \pi}\left[R_{n}^{c s}(r) e^{i l \varphi}\right]^{*} f(r, \varphi) r d r d \varphi e^{-i l \alpha} \\
=M_{n l} e^{-i l \alpha}
\end{gathered}
$$

It can be shown that the PCST of the resulting image are $M_{n l}^{\alpha}=M_{n l} e^{-i l \alpha}$. Considering that $\left|e^{-i l \alpha}\right|=1$. This leads to the result $\left|M_{n l}^{\alpha}\right|=\left|M_{n l}\right|$.

The image function is reconstructed by using inverse transforms. It is universally acknowledged that an approximation of the image function, denoted as $\hat{f}(r, \theta)$, can be given as:

$$
\hat{f}(r, \theta)=\sum_{n=n_{\min }}^{n_{\max }} \sum_{l=l_{\min }}^{l_{\max }} M_{n l} H_{n l}(r, \theta) .
$$

where $n_{\max }, n_{\min }, l_{\max }$, and $l_{\min }$ are the respective maximum and minimum values of PCST, orders and repetitions as specified in (3).

\subsection{The Analysis for the Definition}

In order to solve stability of PHTs, we structure a new radial kernel defined as (5). The same problem does not happen to the radial kernels of PCST when high-order moments are concerned. Furthermore, it focused on cosine or sine through change values of $\mathrm{a}$ and $\mathrm{b}$, making it more suitable for some particular applications. Parameter $\mathrm{a}$ and $\mathrm{b}$ will be discuss by experiments in section 4 . 
The total number of PCST is $\left(1+2 n_{\max }\right)\left(1+2 l_{\max }\right)$, as many as PCET. Moreover, it has more number of moments than PCT and PST, which are $\left(1+n_{\max }\right)\left(1+2 l_{\max }\right)$ and $n_{\max }\left(1+2 l_{\max }\right)$, respectively.

The above analysis shows that PCST is an orthogonal rotation-invariant transform. The proposed method persist the advantages of the PHTs and abandon the disadvantages of the traditional methods. Therefore, PCST will have a wide range of applications.

\section{Experiments}

\subsection{Influence of a and $b$ to PCST}

Here, we show in terms of influence of $a$ and $b$ to PCST. As in [5], the difference between the original image $f(r, \theta)$ and the reconstructed image $\hat{f}(r, \theta)$ is measured using the Root-Mean-Square Error (RMSE).

3 test images with size $128 \times 128$ are selected to get the job done[11]. We plot of the average RMSE values with different $a$ and $b$. It can be observed that the PCSTs with different $a$ and $b$ possess numerical stability even the number of moments is increased up to 8000.It is outstanding than PCET, PCT, PST by experiments later.

Difference of $\mathrm{a}$ and $\mathrm{b}$ to PCSTs can be influence the RMSE values slightly. We selected 4 groups of $\mathrm{a}$ and $\mathrm{b}$ to get the curves, $a^{2}=1.8, b^{2}=0.2, a^{2}=1.5, b^{2}=0.5, a^{2}=0.5, b^{2}=1.5, a^{2}=0.2, b^{2}=1.8$, respectively. The RMSE values are reduced with the increase of moments. According to the values of parameter a, all cases can be divided into two groups. One group for $a^{2} \in(0,1)$ and another group for $a^{2} \in(1,2)$. The second group take advantage of image representation within the number 7000 of moments . In turn, The first group is better than the second group with the number of moments more than 7000. It can be seen from the figure that the curves will reach a very small value when the two group curves intersect at 7000. In fact, the first group is partial to traditional PST and the second group emphasizes PCT. Therefore, PCST has more flexibility for the specific applications though change the value of parameters a and $\mathrm{b}$. Based on the analysis of the curves, We choose $a^{2}=1.5, b^{2}=0.5$ to carry out the following experiments.

\subsection{Image Reconstruction}

In order to verify the performance of our PCST, we present the experimental results for the proposed PCST with traditional ones.

In test experiments, we perform experiments using 30 images from CSIQ [11]. Every database image is of size $256 \times 256$, which cover a variety of topics, such as "native_american", "geckos", "lake", "rushmore", "shroom" etc. CSIQ images have been widely used by the image processing. We resize the 30 images to 128 $\times 128$ pixels.

Let $\mathrm{T}$ be some constant. We have limited the number of moments used in reconstruction based on the following: PCST: $-T \leq n \leq T,-T \leq l \leq T$. PCET: $-T \leq n \leq T,-T \leq l \leq T$. PCT: $0 \leq n \leq T,-T \leq l \leq T$.

PST: $1 \leq n \leq T,-T \leq l \leq T$.

We test four groups: number of moments :61,481,2521,3121,3961,6613,7321,7565 for (a) and (b); 64, 484, 2601, 3136, 4096, 6724, 7396,7569 for (c) and (d), respectively. Same number of moments is used when it comes to PCET and PCST; PCT and PST use another same number of moments. Although there is no way to use the same numbers of moments for four algorithms, they are close to each other.7565 and 7569, only a difference of 4 , for instance. We believe that this way of comparison is feasible and scientific. As more moments are added to the reconstruction process, the reconstructed images get closer to the Original images. The numerical stability of PHTs breaks down when the number of moments is further increased and this problem does not exist in PCST.

In Fig. 1, we show plots of the average RMSE values about 30 images. It can be observed that the proposed PCST have better numerical stability than PHTs, especially superior to PCET. We can easily detect that the RMSE values of PCET are increasing rapidly after 2600 number of moments. As for PCT and PST, here is a similar phenomenon with the increase of the number of moments to 3100 and 4200 , respectively. The curve values of PCST is continue to decline simultaneously. 


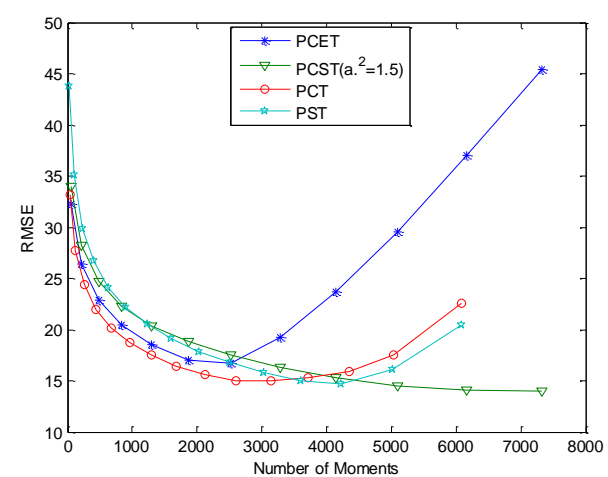

Fig. 1: Average RMSE values of PCSTs and PHTs.

This analysis suggests that the proposed method is effective for stability when high-order moments are concerned in terms of image representation capability.

\subsection{Rotation Invariance}

In order to verify rotation invariance of PCST, an image of plane is resized to $128 \times 128$ pixels and obtained eight rotated versions of it. The value of $n_{\min }$ is fixed to $0, n_{\max }$ is fixed to 3 for PCST. The rotation invariance results are shown in Fig. 2.

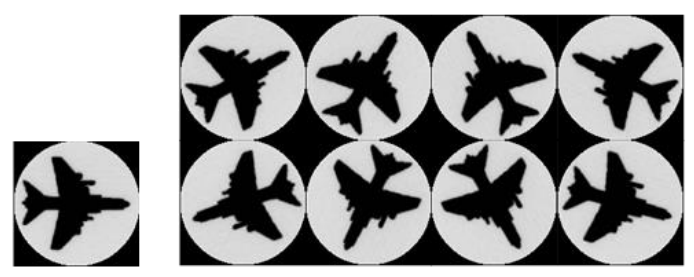

(a)

(b)

Fig. 2: The original image of plane (a) and eight rotated versions of it (b). From left to right (and top to bottom) rotation

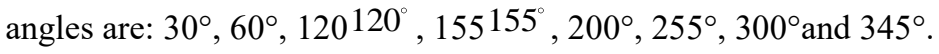

\section{Conclusions}

In this paper, we proposed Polar Cosine-Sine Transform (PCST). It maintains numerical stability superior to PHTs when high-order moments are concerned and possess rotation invariant by a series of experiments. Possible many applications of PCST, such as image retrieval, face detection, etc. The future work is about the fast algorithm of PCST and extended the conventional PCST defined in gray-scale image to color image using the algebra of quaternion.

\section{References}

[1] F. Zernike, Beugungstheorie des schneidenver-fahrens und seiner verbesserten form, der phasenkontrastmethode[J], Physica, 1934,Vol 1, no.5, pp.689-704.

[2] A. B. Bhatia, and E. Wolf, On the circle polynomials of Zernike and related orthogonal sets[J], Mathematical Proceedings of the Cambridge Philosophical Society, 1954, vol.50, no.01, pp.40-48.

[3] Y. Sheng, and L. Shen, Orthogonal FourierCMellin moments for invariant pattern recognition[J], Journal of the Optical Society of America, 1994, vol. 11, no. 6, pp. 1748-1757.

[4] G. Amu, S. Hasi, X. Yang, and Z. Ping, Image analysis by pseudo-Jacobi $(p=4, q=3)$ Fourier moments[J], Applied Optics, 2004,vol. 43, no. 10, pp.2093-2101.

[5] P. -T. Yap, X. Jiang, and A. C. Kot, Two-dimensional polar harmonic transforms for invariant image representation[J], IEEE Trans. Pattern Anal. Mach. Intell, 2010,vol. 32, no. 7, pp. 1259-1270.

[6] T. V. Hoang, and S. Tabbone, Generic polar harmonic transforms for invariant image description[C],Proc. of the 18th IEEE International Conference on Image Processing, Brussels, Belgium, 2011, pp. 845-848.

[7] C. Singh, and R. Upneja, Accuracy and numerical stability of high-order polar harmonic transforms[J], 2012,IET Image Processing, vol. 6, no. 6, pp. 617-626. 
[8] Y. Wo, and J. Xu, Accurate and Fast Harmonic Transform of Polar Coordinates[J], Journal of South China University of Technology (Natural Science Edition), 2012, vol. 40, no. 4, pp. 23-29.

[9] T. V. Hoang, and S. Tabbone, Generic polar harmonic transforms for invariant image representation,Image and Vision Computing, vol. 32, no. 8, pp. 497-509, 2014.

[10] S. Liao, and M. Pawlak, On the Accuracy of Zernike Moments for Image Analysis, IEEE Trans.Pattern Anal. Mach. Intell, vol. 20, no. 12, pp.1358-1364, 1998.

[11] E. C. Larson and D. M. Chandler, “Categorical image quality (CSIQ) database,” [Online], Available: http://vision.okstate.edu/csiq 